\title{
Prognostic Value of Hematogones in Patients with Acute Myeloid Leukemia in First Complete Remission
}

\author{
Mona Hassanein ${ }^{1}$, Rasha Haggag ${ }^{1^{*}}$, Shereen M. El Shorbagy ${ }^{1}$ and Hoda F. Ebian ${ }^{2}$ \\ ${ }^{1}$ Department of Medical Oncology, Faculty of Medicine, Zagazig University, Sharkia, Egypt \\ ${ }^{2}$ Department of Clinical Pathology, Faculty of Medicine, Zagazig University, Sharkia, Egypt
}

*Corresponding author: Rasha Haggag, Department of Medical Oncology, Faculty of Medicine, Zagazig University, Sharkia/EG, Egypt, Tel: +2011 51600020; E-mail: dr_rmmh@yahoo.com

Received date: Jul 19, 2015, Accepted date: Oct 30, 2015, Publication date: Nov 03, 2015

Copyright: (C) 2015 Hassanein M, et al. This is an open-access article distributed under the terms of the Creative Commons Attribution License, which permits unrestricted use, distribution, and reproduction in any medium, provided the original author and source are credited.

\begin{abstract}
Background: Hematogons (HGs) are normal bone marrow cells; that may reflect the quality of the bone marrow response to chemotherapy. Many studies have focused on the role of HGs in acute leukemia.

Methods: A total of 65 patients with nonpromyelocytic AML, in first complete remission were enrolled in this study, and four color flow cytometry was used to quantify Hematogones. We identify the HGs detectable group as those who had more than or equal to $0.01 \% \mathrm{HGs}$ in bone marrow aspirated sample.

Results: HGs were detectable in 25 patients' marrow samples, and they were significantly associated with cytogenetic risk $(p=0.01)$. After a median followup of 17.6 months, patients with detectable HGs had better DFS and OS than those with undetectable levels ( $p=0.013$ and $<0.001$; respectively) and only 3 patients with detectable HGs in marrow remission samples experience relapse. On multivariate analysis, the $H G \geq 0.01 \%$ is an independent predictive value for DFS ( $p<0.0001)$, and OS $(p<0.007)$, but number of chemotherapy cycles to achieve CR and poor cytogenetic had significant prognostic effect on DFS but not on OS, we can conclude that AML patients in first complete remission with $\mathrm{HGs} \geq 0.01 \%$ have better DFS and OS.
\end{abstract}

Conclusions: We can conclude that AML patients in first complete remission with HGs $\geq 0.01 \%$ have better DFS and OS.

Keywords: Hematogones; AML; Prognosis

\section{Introduction}

Acute myeloid leukemia (AML) patient outcomes remain heterogeneous, and the prognosis of AML patients can be estimated based on disease-related and patient-specific factors, such as karyotype abnormalities [1]. Despite these risk factors, the prognosis of patients within each AML subgroup remains heterogeneous and new prognostic tools are needed to assess the risk of relapse [2].

As Hematogones (HGs) are normal B-lymphocyte precursors that are present in bone marrow of healthy persons [3], their biological significance were in their role in hematopoieses with some considering Hematogones to be undifferentiated hematocytyoblast [4].

Hematogones (HGs) were first described as lymphoid appearing cells that were in the sternal aspirates of children undergoing evaluation for an assortment of neoplastic, congenital, infectious and autoimmune disease [5].

They are reported to occur in large numbers in some healthy infants and young children and in a variety of the disease in adults [6]. More prominent especially in the regeneration phase following chemotherapy or bone marrow transplantation and were frequently increased in patients with cytopenias of various etiology as myelodysplastic syndrome [7]. In which their increase in numbers may cause problem in diagnosis due to their morphological features commonly sharing with neoplastic lymphoblast of ALL and lymphoblastic phase of lymphoma [8].

By flowcytometry, Hematogones (HGs) have been characterized into several stages using CD10, CD 19, CD20, CD22, CD34 and CD38 [9], so HGS are often identified by 4-color flowcytometry with coexpression of CD10-CD19 and low intensity CD45 expression.

In order to evaluate the role of HGS level as a new prognostic tool, we conducted this prospective study in AML patients in complete remission to assess their impact on disease free survival and overall survival.

\section{Patients and Methods}

From January 2012 to April 2013, a total of 65 consecutive responding non-promyelocytic patients with AML in first complete remission [10], were enrolled in this study. Patients were admitted to Medical Oncology Department and Clinical Pathology Department, at Zagazig University Hospitals-Egypt. Informed consent was obtained from each patient, the research was conducted in accordance with the 1964 Helsinki Declaration after approval of Zagazig University Institutional review board (IRB). The patients were classified according to their standardized cytogenetic risk subgroups [11]. Samples were obtained by bone marrow (BM) aspiration after hematologic recovery after chemotherapy. All of the patients were treated with Anthracyclin and Ara-C induction chemotherapy schedules; hematogon was 
extracted after induction chemotherapy from BM samples from patients in CR according to Cheson et al. [10]. Haematological complete remission (HCR) is defined as peripheral blood lacking blasts and $<5 \%$ blasts in BM (according to FAB classification), in addition, no immunophenotypical evidence of residual leukaemia was detected by flow

cytometry. Relapse was defined by flow cytometry as the appearance of circulating leukaemia cells or more than 5\% blasts in BM [12].

\section{Flow cytometric method}

In this prospective study, 4 color flowcytometry was used to quantify Hematogones as a percent of total event in consecutive marrow specimen after hematological recovery after chemotherapy. For the assessment of percent and meanflourcent intensity of Hematogones we used two panels of 4 colour combination that were; CD19 PE, CD10 FITC, CD20 Percp, CD45 APC and CD38PE, CD10FITc, CD22 PerCP, CD45 APC, CD45APC, CD34PE, CD10FIITC, CD33Percp, for each sample up to 300.000 cells were acquired and according to plot of side scatter scale versus CD45 stain our data were visualized. The data were collected using FAcscaliber, Bectou-Dickinson BD, and San Diego, USA). Distinct cell populations (Clusters) were identified based on combination of low intensity CD45 expression and CD10/CD19 expression is indicative of HGs; the sensitivity cut off was $0.01 \%$ [9]. We determine the HG positive group as those had more than or equal to $0.01 \%$ HGs in bone marrow aspirated sample. Each specimen's event clusters were considered positive or negative compared with the degree of fluorescence of the same specimen stained with isotype control antibody.

\section{Statistical analysis}

Disease free survival (DFS) was calculated from complete remission (CR) to disease relapse and overall survival (OS) was calculated from diagnosis till death or last follow up. Survival was plotted with KaplanMeier curves, and the data for the various groups were compared with a log-rank test. Multivariate analysis was performed by Cox model after the proportional hazard assumption was checked. $\mathrm{P}$ value less than 0.05 was considered to be statistically significant. All statistical analysis was performed using IBM SPSS software version 20 (Chicago, IL, USA).

\section{Results}

The baseline characteristics of the 65 patients who were included in the study are summarized in Table 1.

Sixty five non-promyelocytic AML were included of which, 39 were male and 26 were female, with male to female ratio was 1.4:1, with a median age of 47 years (18-65), all of the patients received anthracyclin and ara-c ( 3 and 7 protocol as sandaled treatment for AML patients, 2 and 14 for older patients or poor performance status.

Thirty seven patients (56.9\%) responded after 1 cycle, 26 patients (40.7\%) responded after 2 cycles and only 3 patients (3.1\%) respond after 3 cycles. Two patients underwent allogeneic hematopoietic stem cell transplantation (3\%). Twenty five patients (38.5\%) had detectable HGs in the bone marrow remission samples after induction therapy. The median and mean percentages of total HGs were $0 \%$ (range, $0.00-0.53$ ) and $0.086 \%$ (SD \pm 0.138 ), respectively. With 19 patients $(67 \%)$ of them of less than 60 years. No detectable HGs were found in
40 patients' bone marrow samples (30 patients (75\%) were less than 60 years).

Flow-cytometric analysis of BM specimen of HGs was shown in Figure 1.

Twenty two patients (88\%) with detectable HGs had favorable or intermediate risk cytogenetics, that was significantly different from patients with undetectable HGs, in whom 26 patients (65\%) had favorable or intermediate risk cytogenetics ( $\mathrm{p}=0.01$; Figure 2$)$.

After a median follow-up time of 17.6 months, 38 patients (58.5\%) relapsed, and the median DFS was 14 months, only 3 relapsed patients had positive HGs in marrow remission samples. Death occurred in $29 \%$ of the patients (19/65); median OS was not reached, while mean OS was 21.4 months

Patients with detectable HGs had better OS and DFS than those with undetectable levels ( $\mathrm{p}=0.013$ and $<0.0001$; respectively; Figures 3 and 4).

Univariate analysis indicated that the number of chemotherapy cycles required to achieving complete remission, and the standardized cytogenetic risk subgroups and HGs percentage were independent prognostic factors for DFS and OS (Table 2).

According to multivariate Cox model, the HGs $\geq 0.01 \%$ is an independent predictive value for DFS $(\mathrm{p}<0.0001)$, and OS $(\mathrm{p}<0.007)$. While, number of chemotherapy cycles to achieve $C R$, and poor cytogenetic had significant prognostic effect on DFS but not on OS (Table 2).
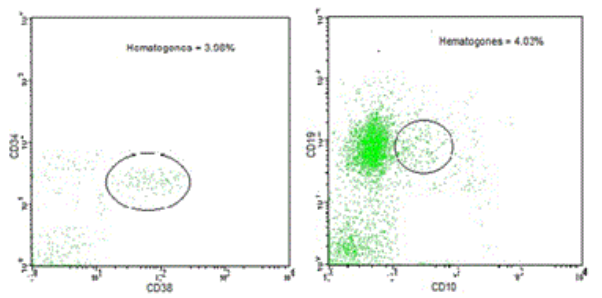

Figure 1: Flowcytometric analysis of $\mathrm{BM}$ specimen Hematogones. A: Hematogones with low $\mathrm{CD} 34$ and $\mathrm{CD} 38$ expression; B: Hematogones with low CD19 low CD10 expression.

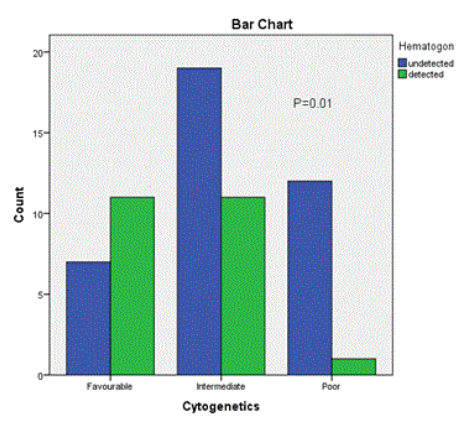

Figure 2: Association between Hematogones and cytogenetics. 
Citation: Hassanein M, Haggag R, El Shorbagy SM, Ebian HF (2015) Prognostic Value of Hematogones in Patients with Acute Myeloid Leukemia in First Complete Remission. J Blood Disord Transfus 6: 319. doi:10.4172/2155-9864.1000319

Page 3 of 6

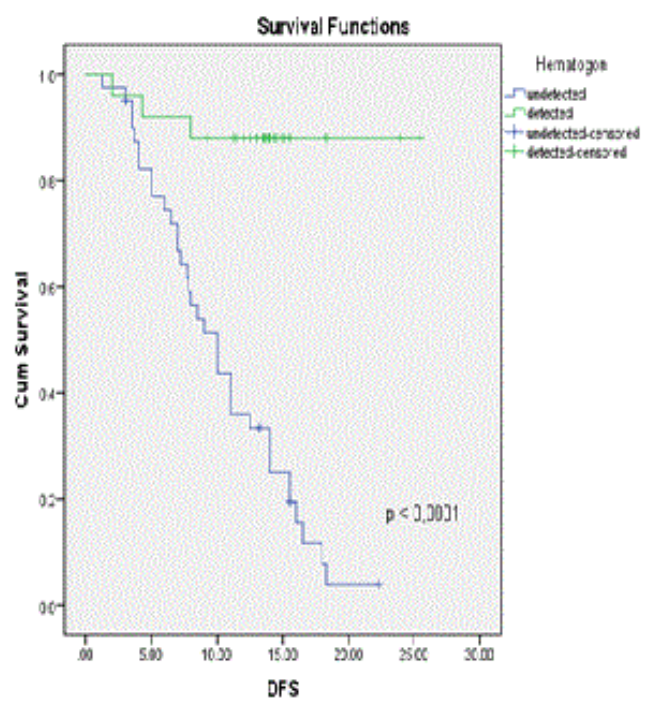

Figure 3: Disease-free survival according to Hematogones status.

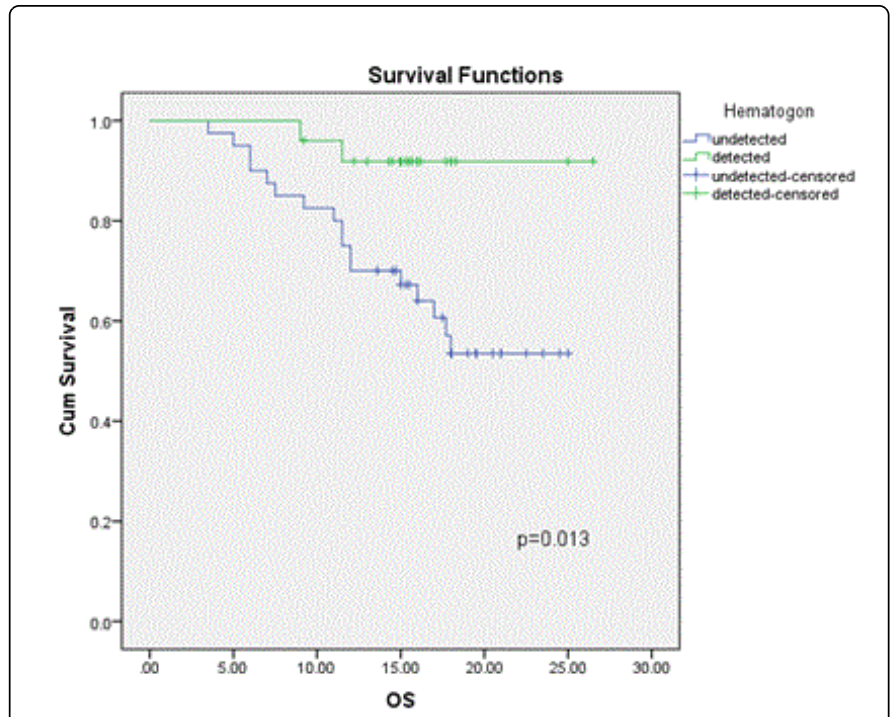

Figure 4: Overall-survival according to Hematogones status.

\begin{tabular}{|c|c|c|}
\hline Patients characteristics & No. & Percentage (\%) \\
\hline \multicolumn{3}{|l|}{ Age } \\
\hline$\leq 60$ years & 49 & $75.4 \%$ \\
\hline$>60$ years & 16 & $24.6 \%$ \\
\hline \multicolumn{3}{|l|}{ Sex } \\
\hline Male & 39 & $60 \%$ \\
\hline Female & 26 & $40 \%$ \\
\hline \multicolumn{3}{|l|}{ FAB Classification } \\
\hline M0 & 3 & $4.6 \%$ \\
\hline M1 & 12 & $18.5 \%$ \\
\hline M2 & 22 & $33.8 \%$ \\
\hline M4 & 14 & $21.5 \%$ \\
\hline M5 & 8 & $12.3 \%$ \\
\hline M6 & 4 & $6.3 \%$ \\
\hline Undifferentiated & 2 & $3.1 \%$ \\
\hline \multicolumn{3}{|l|}{ Cytogenetic } \\
\hline Favorable & 18 & $27.7 \%$ \\
\hline Intermediate & 30 & $46.2 \%$ \\
\hline Poor & 13 & $20 \%$ \\
\hline Failed & 4 & $6.2 \%$ \\
\hline
\end{tabular}


Citation: Hassanein M, Haggag R, El Shorbagy SM, Ebian HF (2015) Prognostic Value of Hematogones in Patients with Acute Myeloid Leukemia in First Complete Remission. J Blood Disord Transfus 6: 319. doi:10.4172/2155-9864.1000319

Page 4 of 6

\begin{tabular}{|l|l|l|}
\hline 1 & 37 & $56.9 \%$ \\
\hline 2 & 26 & $40.7 \%$ \\
\hline 3 & 2 & $3.1 \%$ \\
\hline Hematogons & 2 & $38.5 \%$ \\
\hline$>0.01$ & 25 & $61.5 \%$ \\
\hline$<0.01$ & 40 & \\
\hline
\end{tabular}

Table 1: Shows patients characteristics.

\begin{tabular}{|c|c|c|c|c|}
\hline \multicolumn{5}{|c|}{ DFS } \\
\hline \multirow[t]{2}{*}{ Prognostic factors } & \multirow[t]{2}{*}{ Univariate analysis } & \multicolumn{3}{|c|}{ Multivariate analysis } \\
\hline & & HR & $95 \% \mathrm{Cl}$ & $\mathbf{P}$ \\
\hline Age & 0.297 & 0.68 & $0.325-1.42$ & 0.3 \\
\hline Hematogons $\geq 0.01 \%$ & $<0.001$ & 2.3 & $3.12-33.3$ & $<0.0001$ \\
\hline Leucoytosis & 0.166 & 0.58 & $0.29-1.16$ & 0.125 \\
\hline No. of chemotherapy cycles to achieve CR & $<0.0001$ & 0.318 & $0.07-1.4$ & $<0.0001$ \\
\hline \multicolumn{5}{|l|}{ Cytogenetics } \\
\hline Favorable & \multirow[t]{3}{*}{$<0.0001$} & 0.799 & $0.245-2.610$ & 0.710 \\
\hline Intermediate & & 1.225 & $0.400-3.752$ & 0.722 \\
\hline Poor & & 6.024 & $1.774-20.4$ & 0.004 \\
\hline \multicolumn{5}{|c|}{ os } \\
\hline \multirow[t]{2}{*}{ Prognostic factors } & \multirow[t]{2}{*}{ Univariate analysis } & \multicolumn{3}{|c|}{ Multivariate analysis } \\
\hline & & HR & $95 \% \mathrm{Cl}$ & $\mathbf{P}$ \\
\hline Age & 0.152 & 0.513 & $0.2-1.3$ & 0.162 \\
\hline Hematogons $\geq 0.01 \%$ & 0.013 & 1.6 & $1.2-22.9$ & 0.007 \\
\hline Leucoytosis & 0.136 & 2.134 & $0.766-5.944$ & 0.147 \\
\hline No. of chemotherapy cycles to achieve CR & 0.002 & 0.218 & $0.017-2.790$ & 0.241 \\
\hline \multicolumn{5}{|l|}{ Cytogenetics } \\
\hline Favorable & \multirow[t]{3}{*}{$<0.0001$} & 0.605 & $0.68-5.375$ & 0.652 \\
\hline Intermediate & & 1.468 & $0.243-8.868$ & 0.676 \\
\hline Poor & & 3.741 & $0.653-21.422$ & 0.138 \\
\hline
\end{tabular}

Table 2: Impact of prognostic factors on disease-free survival and overall survival.

\section{Discussion}

Despite high remission rate in acute myeloid leukemia (AML) after chemotherapy, relapse of the underlying disease remains a major challenge and one of the most frequent causes of treatment failure. The major prognostic parameters with regard to relapse of AML are the response to induction chemotherapy and the genetic abnormalities of the malignant cells [13]. In our study, we assessed if monitoring bone marrow HGs in AML patients following induction chemotherapy can be used as a prognostic factor. We used four color flow-cytometry for detection of HGs. HGs were detected in $38.64 \%$ of our patients compared to $46 \%$ reported by Sylvain et al. [2] and $57.7 \%$ by Zheng et al. [14]. This difference may be due to larger number of patients included in their studies. 
Page 5 of 6

Although the quantity of B-cell precursors decreases with age, HGs were identified in $37.5 \%$ of our patients older than 60 years, median age of 47 years (18-65), with no statistically significant differences between the age groups $(\mathrm{P}>0.5)$. This is matched with Kern et al. [15] and Sylvain et al. [2].

In discordance with our results, Zheng et al. [14] reported a statistically significant decrease in HGs was observed when comparing older age categories. This difference may be due to including pediatric patients and only 92/292 samples were collected from patients older than 50 years.

Also we did not find significant association between HGs percentages and gender or AML subtypes ( $\mathrm{p}=0.3$ and 0.4 , respectively) which is similar to results of Zheng et al. [14].

In our study there was significant association between detectable HGs percentages and cytogenetic risk $(\mathrm{p}=0.01)$ which is in agreement with study by Zheng et al. [14] and Pereira et al. [16].

Only 3 of our patients (12\%) with detectable HGs in marrow remission samples had relapse. However, Zheng et al. [14] reported that $36.7 \%$ with detectable HGs experienced relapse with Significant differences in HGs percentages were detected between complete remission and relapse categories $(\mathrm{P}=0.028)$.

After a median follow-up time of 17.6 months, univariate and multivariate analysis of prognostic factors indicated that HGs status is a prognostic factor for both DFS and OS.

Zheng et al. [14] reported a median follow-up time was 28 months for all patients, patients who had more than $0.1 \%$ of HGs in their bone marrow had a higher median leukaemia-free survival (LFS) (24.75 vs. 9.5, $\mathrm{P}<0.001)$ and $\mathrm{OS}(25.3$ vs. $10.75, \mathrm{P}<0.001)$, compared with patients who had $<0.1 \%$ of HGs in their bone marrow.

Because of significant differences in HCR (Hematological Complete Remission) between the group containing $>0.1 \%$ of HGs and those containing $<0.1 \%$ of HGs, they chose $0.1 \%$ of HGs as the cut-off level to investigate survival differences $(0.1 \%$ as a cutoff point of HGs percentage is different from ours and previously reported data which displayed that HGs level more than $0.01 \%$ was an independent prognostic factor for AML patients after the first induction chemotherapy), Also through their univariate analysis; HGs, risk stratification and leucocytosis at the time of diagnosis all contributed to OS and LFS, whereas age is unrelated to both. Chemotherapy cycle is one prognostic factor for LFS but not for OS. Cox analysis indicated that HG presence was independently associated with a longer LFS but was not associated with OS.

Also Sylvain et al. [2] reported patients who had HG levels more than $0.01 \%$ had a significantly better median LFS (29.2 vs 11.7 months; $\mathrm{P}=0.001$ ) and overall survival

(Not reached vs. 23.5 months; $\mathrm{P}=0.011$ ). According to Cox analysis, a HG level more than $0.01 \%$ was an independent predictor of LFS $(\mathrm{HR}=0.5 ; 95 \%$ confidence interval, $0.28-0.90 ; \mathrm{P}<0.03)$ when age, leucocytosis, the number of chemotherapy cycles, and the standardized cytogenetic and molecular risk subgroups were controlled for. Cox analysis indicated that HG presence was independently associated with a longer LFS but not with OS.

This difference of results may be due to larger number of patients included in their studies or due to different patient's characteristics.

\section{Conclusion}

We found that HGs more than $0.01 \%$ in bone marrow after induction therapy was associated with decreased risk of relapse and increased survival, and usage of 4 color flow cytometry for detection of HGs is simple, rapid and suitable method for clinical screening mainly in developing countries.

\section{Conflict of Interest}

The authors indicated no potential conflict of interest.

\section{References}

1. Grimwade D, Walker H, Oliver F, Wheatley K, Harrison C, et al. (1998) The importance of diagnostic cytogenetics on outcome in AML: analysis of 612 patients entered into the MRC AML 10 trial. The Medical Research Council Adult and Children's Leukaemia Working Parties. Blood 92: 2322-2333.

2. Chantepie SP, Salaün V, Parienti JJ, Truquet F, Macro M, et al. (2011) Hematogones: a new prognostic factor for acute myeloblastic leukemia. Blood 117: 1315-1318.

3. Muehleck SD, McKenna RW, Gale PF, Brunning RD (1983) Terminal deoxynucleotidyl transferase (TdT)- positive cells in bone marrow in the absence of hematologic malignancy. Am J Clin Pathol 79: 277-284.

4. Maximow AA, Bloom W (1930) A text book of bistology. Philadelphia, W.B Saunders company.

5. Vogel P, Erf LN, Rosental N (1937) Hematological observations on bone marrow obtained by sternal puncture. Am J Clin Pathol 7: 436-447.

6. Klein U, Tu Y, Stolovitzky GA, Mattioli M, Cattoretti G, et al. (2001) Gene expression profiling of $\mathrm{B}$ cell chronic lymphocytic leukemia reveals a homogeneous phenotype related to memory B cells. J Exp Med 194: 1625-1638.

7. Koca E, Buyukasik Y, Cetiner D, Yilmaz R, Sayinalp N, et al. (2008) Copper deficiency with increased hematogones mimicking refractory anemia with excess blasts. Leuk Res 32: 495-499.

8. Caldwell CW, Poje E, Helikson MA (1991) B-cell precursors in normal pediatric bone marrow. Am J Clin Pathol 95: 816-823.

9. McKenna RW, Washington LT, Aquino DB, Picker LJ, Kroft SH (2001) Immunophenotypic analysis of hematogones (B-lymphocyte precursors) in 662 consecutive bone marrow specimens by 4-color flow cytometry. Blood 98: 2498-2507.

10. Cheson BD, Bennett JM, Kopecky KJ, Büchner T, Willman CL, et al. (2003) Revised recommendations of the International Working Group for Diagnosis, Standardization of Response Criteria, Treatment Outcomes, and Reporting Standards for Therapeutic Trials in Acute Myeloid Leukemia. J Clin Oncol 21: 4642-4649.

11. Döhner H, Estey EH, Amadori S, Appelbaum FR, Büchner T, et al. (2010) Diagnosis and management of acute myeloid leukemia in adults: recommendations from an international expert panel, on behalf of the European LeukemiaNet. Blood 115: 453-474.

12. Babusíková O, Zelezníková T, Kirschnerová G, Kankuri E (2008) Hematogones in acute leukemia during and after therapy. Leuk Lymphoma 49: 1935-1944.

13. Ribeiro E, Matarraz Sudón S, de Santiago M, Lima CS, Metze K, et al. (2006) Maturation-associated immunophenotypic abnormalities in bone marrow B-lymphocytes in myelodysplastic syndromes. Leuk Res 30: 9-16.

14. Zheng J, Du W, Yao J, You Y, Li W, et al. (2013) Analysis of hematogones in bone marrow from acute myeloid leukaemia cases posttherapy. Eur J Clin Invest 43: 1140-1146.

15. Kern W, Danhauser-Riedl S, Ratei R, Schnittger S, Schoch C, et al. (2003) Detection of minimal residual disease in unselected patients with acute myeloid leukemia using multiparameter flow cytometry for definition of leukemia-associated immunophenotypes and determination of their frequencies in normal bone marrow. Haematologica 88: 646-653. 
Citation: Hassanein M, Haggag R, El Shorbagy SM, Ebian HF (2015) Prognostic Value of Hematogones in Patients with Acute Myeloid Leukemia in First Complete Remission. J Blood Disord Transfus 6: 319. doi:10.4172/2155-9864.1000319

Page 6 of 6

16. Pereira D, Chacim SP, Mesquite E, Espirito-Santo A, Moreira I, et al. (2012) Prognostic Impact of High Hematogon in Acute Myeloid
Leukemia. Poster presentation at the American Society of hematology, Atlanta GA. 